\title{
The structure of solution sets of fuzzy relation equations
}

\author{
Xiaobing Qu, ${ }^{1}$ Feng Sun, ${ }^{1}$ Tianfei Wang, ${ }^{1}$ Qingquan Xiong ${ }^{2}$ \\ ${ }^{1}$ College of Mathematics and Information Science, Leshan Normal University, \\ Leshan, Sichuan 614004, People's Republic of China \\ ${ }^{2}$ College of Mathematics and Software Science, Sichuan Normal University, \\ Chengdu, Sichuan 610066, People's Republic of China
}

\begin{abstract}
In this paper, we consider the structure of solution sets of fuzzy relation equations over complete Boolean algebras. We show that each solution of a system of fuzzy relation equations can be represented by a linear combination of a special solution of its and some certain solutions of the homogeneous equations associated with the system.
\end{abstract}

Keywords: Fuzzy relation equations; Complete Boolean algebra; Structure of solution set

\section{Introduction}

System of fuzzy relation equations is an importan$\mathrm{t}$ topic in fuzzy set theory. In 1976, Sanchez [21] first introduced fuzzy relation equations with supinf composition in complete Brouwerian lattices. Since then, many authors investigated the methods for solving fuzzy relation equations with different composite operators over various special Brouwerian lattices. Among them, for finite fuzzy relation equations with sup-inf composition, Higashi et al. [11] showed that the solution set can be determined by minimal solutions and the greatest solution in the linear lattice $[0,1]$. Zhao [27] determined its entirely solution set in complete and completely distributive lattices. De Baets [4] determined all minimal solutions and Wang [24] gave a formula of the number of minimal solutions under the condition that all elements of its righthand side have irredundant finite join-irreducible decompositions. And many other works about solving fuzzy relation equations were published (see $[1,5,8,10,12,13,14,15,16,17,19,20,22,23])$. Compared with the linear algebraic systems, the main difference with fuzzy relation equations is the operations and the fundamental domain. Recently, there are some ideas which learn from linear algebraic systems for studying fuzzy relation equations. In 2004, Perfilieva [18] introduced the concept of semi-linear space based on BL-algebra. Di Nola et al. [7] introduced the concepts of semilinear space, basis and linear independent based on MV-algebra, and got to connect the semi-linear space and systems of fuzzy relation equations. Wang and Zhao[26] described the solution set of systems of fuzzy relation equations through $n$-dimensional vectors over bounded Brouwerian lattices.

A generic version of fuzzy relation equation arises in the form

$$
A \odot \mathbf{x}=\mathbf{b}
$$

or

$$
\bigvee_{j \in J}\left(a_{i j} \wedge x_{j}\right)=b_{i}, \text { for all } i \in I,
$$

where $A=\left(a_{i j}\right)_{(i, j) \in I \times J}$ and $\mathbf{b}=\left(b_{i}\right)_{i \in I}^{T}$ are known ( $I$ and $J$ are index sets with $I=\{1,2, \cdots, m\}$ and $J=\{1,2, \cdots, n\}), \mathbf{x}=\left(x_{j}\right)_{j \in J}^{T}$ is unknown, $\odot$ denotes the sup-inf composite operation, and all $b_{i}, x_{j}, a_{i j}$ 's are in a complete Brouwerian lattice $L$. An $\mathbf{x}$ which satisfies Eq.(1) is called a solution of (1), the solution set of Eq.(1) is denoted by $\mathcal{X}=\{\mathbf{x}: A \odot \mathbf{x}=\mathbf{b}\}$. If $\mathcal{X} \neq \emptyset$, the greatest element of $\mathcal{X}$ is denoted by $\mathbf{x}^{*}=\left(x_{j}^{*}\right)_{j \in J}^{T}$, and we denote $\mathcal{X}^{0}=\{\mathbf{x} \in \mathcal{X}: \mathbf{x}$ is a minimal element of $\mathcal{X}\}$. The following equation is called the homogeneous equations associated with Eq.(1)

$$
A \odot \mathbf{x}=\mathbf{0},
$$

where $\mathbf{0}=(0,0, \cdots, 0)^{T}$ is a zero vector. $\mathcal{Y}$ and $\mathbf{y}^{*}$ denote the solution set and the greatest solution of Eq.(2), respectively.

In this paper, we consider the structure of Eq.(1) over a special complete Brouwerian lattice, that is, a complete Boolean algebra $(L, \vee, \wedge, 0,1)$. We will describe the solution set of Eq.(1) by a linear combination of a special solution of its and some certain solutions of Eq.(2). This paper is organized as follows. Section 2 presents some preliminary information which includes fuzzy relation equations and lattices. Section 3 gives some properties of elements in complete Boolean algebras. In section 4, we describe the structure of solution sets of fuzzy relation equations. Conclusions are given in section 5 .

\section{Preliminaries}

In this section, we give some definitions and previous results for the sake of convenience.

Definition 2.1 [2] A lattice $L$ is Brouwerian if for any pair of elements $a, b \in L$, the greatest element 
$x \in L$, denoted by $a \alpha b$, satisfying the inequality $a \wedge$ $x \leqslant b$ exists.

Definition 2.2 [3, 23] Let $L$ be a complete lattice and $a \in L$. If $T$ is a finite subset of $L$ and $S$ ( $f$ nite or infinite) is a subset of $L$, the representation $a=\bigvee T$ is called an irredundant finite join decomposition of a if $a \neq \bigvee_{t \in T}(T-\{t\})$ for each $t \in T$, and the representation $a=\bigvee S$ is called a minimal join decomposition of $a$ if $a \neq q \vee \bigvee_{s \in S, s \neq p} s$ for each $p \in S$ and for any $q<p$.

Let $L$ be a complete lattice. For each $a \in L$, denote $\mathcal{M}_{a}=\{M \subseteq L: a=\vee M$ is a minimal join decomposition of $a\}$. It is clear that $\{a\} \in \mathcal{M}_{a}$.

Definition 2.3 [3] Let $L$ be a lattice with a least element 0 and a greatest element 1 . A complement of an element $a \in L$ is an element $b \in L$ such that $a \wedge b=0$ and $a \vee b=1$. We write $b=a^{\prime}$. $L$ is called complemented if each of its elements has a complement, and $L$ is relatively complemented if the quotient sublattice $a / b=\{x \in L: b \leqslant x \leqslant a\}$ is complemented for each pair of elements $a>b$ in L. A complemented, distributive lattice is called a Boolean algebra. A complete Boolean algebra $L$ is infinitely distributive; i.e., if $a \in L$ and $S$ is a subset of $L$, then $a \wedge \bigvee S=\bigvee_{s \in S}(a \wedge s)$

Let $L$ be a complete relatively complemented lattice, $a \in L$ and $a \neq 0, b \leqslant a$, we denote the complement of $b$ in the sublattice $a / 0$ by $b_{a}^{\prime}$.

Lemma 2.1 [3] Every complemented modular lattice is relatively complemented. In particular, Boolean algebras are relatively complemented.

Lemma 2.2 [2] Let $L$ be a Boolean algebra. Then $(a \wedge b)^{\prime}=a^{\prime} \vee b^{\prime}$ for all $a, b \in L$.

Lemma 2.3 [9] Let $L$ be a Boolean algebra. Then $a \alpha b=a^{\prime} \vee b$ for all $a, b \in L$.

Lemma 2.4 [21] If $\mathcal{X} \neq \emptyset$, then $\mathbf{x}^{*}=$ $\left(\bigwedge_{i \in I}\left(a_{i j} \alpha b_{i}\right)\right)_{j \in J}^{T}$ is the greatest element of $\mathcal{X}$. If $\mathbf{x}_{1}, \mathbf{x}_{2} \in \mathcal{X}$ and $\mathbf{x}_{1} \leqslant \mathbf{x}_{2}$, then $\mathbf{x} \in \mathcal{X}$ for any $\mathbf{x}$ such that $\mathbf{x}_{1} \leqslant \mathbf{x} \leqslant \mathbf{x}_{2}$.

Lemma 2.5 [27] For equation $\bigvee_{j \in J}\left(a_{j} \wedge x_{j}\right)=b$, $\mathcal{X} \neq \emptyset$ if and only if $b \leqslant \bigvee_{j \in J} a_{j}$.

Remark 2.1 For equation $\bigvee_{j \in J}\left(a_{j} \wedge x_{j}\right)=b$, by Lemma 2.5, $\mathcal{X} \neq \emptyset$ if and only if $\mathbf{x}=(b)_{j \in J} \in \mathcal{X}$.

Lemma 2.6 [25] For equation $\bigvee_{j \in J}\left(a_{j} \wedge x_{j}\right)=b$, if $\mathbf{x}_{*}=\left(x_{j *}\right)_{j \in J}^{T}$ is a minimal element of $\mathcal{X}$, then $b=\bigvee_{j \in J} x_{j *}$, and for all $j \in J, x_{j *} \leqslant a_{j}$.

\section{Properties of elements in complete Boolean algebras}

In this section, we focus on discussing the properties of elements in complete Boolean algebras. In the rest of this paper, unless otherwise specified, we always assume that $L$ is a complete Boolean algebra with the least element 0 and the greatest element 1. For each $a \in L, a \neq 0$, denote $D(a)=\{x \leqslant a$ : there is some element $y<a$ such that $x \vee y=a\}$.

Theorem 3.1 Let $a, b \in L$, and $a \leqslant b$. Then $a_{b}^{\prime}=a^{\prime} \wedge b$.

Proof. Since $a \vee a^{\prime}=1$ and $a \wedge a^{\prime}=0$, it follows that $a \vee\left(a^{\prime} \wedge b\right)=\left(a \vee a^{\prime}\right) \wedge(a \vee b)=1 \wedge b=b$, and $a \wedge\left(a^{\prime} \wedge b\right)=\left(a \wedge a^{\prime}\right) \wedge b=0 \wedge b=0$. Then $a_{b}^{\prime}=a^{\prime} \wedge b$.

Theorem 3.2 Let $a \in L$. Then for every $b \in$ $D(a)$, there is some $M \in \mathcal{M}_{a}$ such that $b \in M$.

Proof. If $b=a$, then $b \in\{a\}$ and $\{a\} \in \mathcal{M}_{a}$. If $b<a$, then there is $b_{a}^{\prime}$ such that $b \vee b_{a}^{\prime}=a$ and $b \wedge b_{a}^{\prime}=0$. We infer that $M=\left\{b, b_{a}^{\prime}\right\} \in \mathcal{M}_{a}$. If there exists $c<b$ such that $c \vee b_{a}^{\prime}=a$, then $c \wedge b_{a}^{\prime}=0$ since $b \wedge b_{a}^{\prime}=0$. This implies that $c$ is a relatively complement of $b_{a}^{\prime}$. So $b_{a}^{\prime}$ has two different complements, a contradiction. Similarly, there is no element $c<b_{a}^{\prime}$ such that $b \vee c=a$. Therefore, by Definition $2.2, M=\left\{b, b_{a}^{\prime}\right\} \in \mathcal{M}_{a}$.

In the following two theorems, the index set $J$ is defined as previous, that is, $J=\{1,2, \cdots, n\}$.

Theorem 3.3 If $a \in L$ and $a=\bigvee_{j \in J} t_{j}$ is an irredundant finite join decomposition of a, then there exists $M=\left\{s_{j} \in L: j \in J\right\} \in \mathcal{M}_{a}$ such that $s_{j} \leqslant t_{j}$ for all $j \in J$.

Proof. Choose any $k \in J$, let $a_{k}=\bigvee_{j \in J, j \neq k} t_{j}$, and $S=\left\{x \in L: x \vee a_{k}=a\right\}$. Certainly, $S \neq \emptyset$ since $t_{k} \in S$. Because of the upper continuity of $L$, there is a minimal element $s_{k} \in S$ (With the distributivity, in fact, $s_{k}$ is the least element of $S$ ), that is, $a=s_{k} \vee a_{k}$ and $a \neq x \vee a_{k}$ for any $x<$ $s_{k}$. Next, choose any $k_{1} \in J-\{k\}$ and let $a_{k_{1}}=$ $s_{k} \vee \bigvee_{j \in J, j \neq k, k_{1}} t_{j}$. It is similar that there is an element $s_{k_{1}} \in L$ such that $a=s_{k_{1}} \vee s_{k} \vee a_{k_{1}}$ and $a \neq x \vee s_{k} \vee a_{k_{1}}$ for any $x<s_{k_{1}}$. Continuing in this way we can obtain a set $M=\left\{s_{j} \in L: j \in J\right\} \in$ $\mathcal{M}_{a}$ such that $s_{j} \leqslant t_{j}$ for all $j \in J$.

Theorem 3.4 Let $a, b \in L, b<a$ and $b \in D(a)$. If $a=b \vee \bigvee_{j \in J} t_{j}$, then there exist elements $s_{j} \leqslant t_{j}$, $j \in J$ such that $a=b \vee \bigvee_{j \in J} s_{j}$ and $a \neq \bigvee_{j \in J} s_{j}$. Moreover, there is an element $M \in \mathcal{M}_{a}$ such that $b \in M$ and for every $u \in M, u \neq b$, there exists some $t_{j}$ such that $u \leqslant t_{j}$.

Proof. Assume that $b<a$ and $b \in D(a), b_{a}^{\prime}$ is the relatively complement of $b$ in sublattice $a / 0$. Then $b \vee b_{a}^{\prime}=a$. Let $s_{j}=t_{j} \wedge b_{a}^{\prime}, j \in J$. Since $a=b \vee b_{a}^{\prime}$ 
and $a=b \vee \bigvee_{j \in J} t_{j}$, we have $a=b \vee\left(b_{a}^{\prime} \wedge \bigvee_{j \in J} t_{j}\right)=$ $b \vee \bigvee_{j \in J}\left(b_{a}^{\prime} \wedge t_{j}\right)=b \vee \bigvee_{j \in J} s_{j}$. In addition, with the fact $b_{a}^{\prime}<a$, we have $\bigvee_{j \in J} s_{j}=\bigvee_{j \in J}\left(b_{a}^{\prime} \wedge t_{j}\right) \leqslant$ $b_{a}^{\prime}<a$. Hence $a \neq \bigvee_{j \in J} s_{j}$. Now removing the redundant elements from the representation $a=b \vee$ $\bigvee_{j \in J} s_{j}$, we can get an irredundant represention $a=$ $b \bigvee \bigvee_{j \in J^{\prime}} s_{j}$ and $a \neq b \vee \bigvee_{j \in J^{\prime}, j \neq k} s_{j}$ for each $k \in J^{\prime}$, where $J^{\prime} \subseteq J$. Similar to the proof of Theorem 3.2, there is no element $c<b$ such that $a=c \vee \bigvee \bigvee_{j \in J^{\prime}} s_{j}$. Furthermore, according to Theorem 3.3, there are elements $u_{j} \in L, j \in J^{\prime}$, and $u_{j} \leqslant s_{j}$ for all $j \in J^{\prime}$ such that $M=\left\{u_{j} \in L: j \in J^{\prime}\right\} \cup\{b\} \in \mathcal{M}_{a}$. It is clear that $u_{j} \leqslant s_{j} \leqslant t_{j}$ for all $j \in J^{\prime}$, the proof is complete.

\section{Structure of solution set of fuzzy relation equations}

In this section, we will discuss the structure of solution set of Eq.(1) similar to linear algebraic systems. In the following, for the sake of convenience, we sometimes denote $a \vee b$ and $a \wedge b$ by $a+b$ and $a b$, respectively.

For Eq.(2), it is clear that $\mathcal{Y} \neq \emptyset$ since $\mathbf{0}_{n \times 1}=$ $(0,0, \cdots, 0)^{T} \in \mathcal{Y}$, the greatest solution is $\mathbf{y}^{*}=$ $\left(y_{j}^{*}\right)_{j \in J}^{T}=\left(\bigwedge_{i \in I} a_{i j}^{\prime}\right)_{j \in J}^{T}=\left(\left(\bigvee_{i \in I} a_{i j}\right)^{\prime}\right)_{j \in J}^{T}$, and $\mathbf{y}^{*} \leqslant \mathbf{x}^{*}$. Now for each $k \in J$, define $\mathbf{e}_{k}=\left(y_{j}\right)_{j \in J}^{T}$ with $y_{k}=\left(\bigvee_{i \in I} a_{i j}\right)^{\prime}$ and $y_{j}=0$ if $j \neq k$. Then $\mathbf{e}_{k} \in \mathcal{Y}$ for all $k \in J$, and for any $\mathbf{y}=\left(y_{j}\right)_{j \in J}^{T} \in \mathcal{Y}$, we have $\mathbf{y}=y_{1} \mathbf{e}_{1}+y_{2} \mathbf{e}_{2}+\cdots+y_{n} \mathbf{e}_{n}$. Therefore, $\mathcal{Y}=\left\{y_{1} \mathbf{e}_{1}+y_{2} \mathbf{e}_{2}+\cdots+y_{n} \mathbf{e}_{n}: y_{j} \leqslant y_{j}^{*}\right.$ for all $j \in$ $J\}$.

Now we consider the structure of solution set of Eq.(1).

Theorem 4.1 If $\mathbf{x} \in \mathcal{X}$ and $\mathbf{y} \in \mathcal{Y}$, then $\mathbf{x} \vee \mathbf{y} \in \mathcal{X}$ and $\mathbf{x} \wedge \mathbf{y} \in \mathcal{Y}$.

Proof. Suppose that $\mathbf{x}=\left(x_{j}\right)_{j \in J}^{T} \in \mathcal{X}$ and $\mathbf{y}=\left(y_{j}\right)_{j \in J}^{T} \in \mathcal{Y}$. Then $\bigvee_{j \in J}\left(a_{i j} \wedge x_{j}\right)=b_{i}$ and $\bigvee_{j \in J}\left(a_{i j} \wedge y_{j}\right)=0$ for all $i \in I$. Thus for all $i \in I$, $\bigvee_{j \in J}\left(a_{i j} \wedge\left(x_{j} \vee y_{j}\right)\right)=\bigvee_{j \in J}\left(\left(a_{i j} \wedge x_{j}\right) \vee\left(a_{i j} \wedge y_{j}\right)\right)=$ $\bigvee_{j \in J}\left(a_{i j} \wedge x_{j}\right) \vee \bigvee_{j \in J}\left(a_{i j} \wedge y_{j}\right)=b_{i} \vee 0=b_{i}$, and $\bigvee_{j \in J}\left(a_{i j} \wedge\left(x_{j} \wedge y_{j}\right)\right) \leqslant \bigvee_{j \in J}\left(a_{i j} \wedge y_{j}\right)=0$. This implies that $\bigvee_{j \in J}\left(a_{i j} \wedge\left(x_{j} \wedge y_{j}\right)\right)=0$. Therefore, $\mathbf{x} \vee \mathbf{y} \in \mathcal{X}$ and $\mathbf{x} \wedge \mathbf{y} \in \mathcal{Y}$.

Theorem 4.2 For any $\mathrm{x} \in \mathcal{X}$, there is a solution $\mathbf{w} \in \mathcal{X}$ such that $\mathbf{x}=\mathbf{w} \vee\left(\mathbf{y}^{*} \wedge \mathbf{x}\right)$.

Proof. Suppose that $\mathbf{x}=\left(x_{j}\right)_{j \in J}^{T} \in \mathcal{X}$. Then $\mathbf{y}^{*} \wedge \mathbf{x}=\left(\left(\bigvee_{i \in I} a_{i j}\right)^{\prime} \wedge x_{j}\right)_{j \in J}^{T}$. Let $\mathbf{w}=\left(w_{j}\right)_{j \in J}^{T}$, where $w_{j}=\left(\left(\bigvee_{i \in I} a_{i j}\right)^{\prime} \wedge x_{j}\right)_{x_{j}}^{\prime}$ for all $j \in J$. Since $\left(\left(\bigvee_{i \in I} a_{i j}\right)^{\prime} \wedge x_{j}\right)_{x_{j}}^{\prime}=\left(\left(\bigvee_{i \in I} a_{i j}\right)^{\prime} \wedge x_{j}\right)^{\prime} \wedge x_{j}=$ $\left(\left(\bigvee_{i \in I} a_{i j}\right) \vee x_{j}^{\prime}\right) \wedge x_{j}=\left(\left(\bigvee_{i \in I} a_{i j}\right) \wedge x_{j}\right) \vee\left(x_{j} \wedge x_{j}^{\prime}\right)=$ $\left(\left(\bigvee_{i \in I} a_{i j}\right) \wedge x_{j}\right) \vee 0=\left(\bigvee_{i \in I} a_{i j}\right) \wedge x_{j}$, we have $\bigvee_{j \in J}\left(a_{i j} \wedge w_{j}\right)=\bigvee_{j \in J}\left(a_{i j} \wedge\left(\bigvee_{i \in I} a_{i j}\right) \wedge x_{j}\right)=$ $\bigvee_{j \in J}\left(a_{i j} \wedge x_{j}\right)=b_{i}$ for all $i \in I$. Thus $\mathbf{w} \in \mathcal{X}$. It can be easily shown that $\mathbf{x}=\mathbf{w} \vee\left(\mathbf{y}^{*} \wedge \mathbf{x}\right)$.
Remark 4.1 For any $\mathbf{x}=\left(x_{j}\right)_{j \in J}^{T} \in \mathcal{X}$, by Theorem $4.1, \mathbf{y}^{*} \wedge \mathbf{x}=\left(y_{j}\right)_{j \in J}^{T} \in \mathcal{Y}$ holds. It follows that $\mathbf{y}^{*} \wedge \mathbf{x}=y_{1} \mathbf{e}_{1}+y_{2} \mathbf{e}_{2}+\cdots+y_{n} \mathbf{e}_{n}$. Consequently, according to Theorem 4.2, there is a special solution $\mathbf{w} \in \mathcal{X}$ such that $\mathbf{x}=\mathbf{w}+y_{1} \mathbf{e}_{1}+y_{2} \mathbf{e}_{2}+\cdots+y_{n} \mathbf{e}_{n}$. But if $\mathbf{x}_{1}, \mathbf{x}_{2} \in \mathcal{X}$, and $\mathbf{x}_{1} \neq \mathbf{x}_{2}$, then the special solution $\mathbf{w} \in \mathcal{X}$ need not be the same one.

Let $\mathbf{y}^{*^{\prime}}=\left(y_{j}^{*^{\prime}}\right)_{j \in J}^{T}=\left(\bigvee_{i \in I} a_{i j}\right)_{j \in J}^{T}$, and $\mathbf{w}_{0}=$ $\mathbf{x}^{*} \wedge \mathbf{y}^{*^{\prime}}$. Since for all $i \in I, \bigvee_{j \in J}\left(a_{i j} \wedge\left(x_{j}^{*} \wedge\right.\right.$ $\left.\left.\vee_{i \in I} a_{i j}\right)\right)=\bigvee_{j \in J}\left(a_{i j} \wedge x_{j}^{*}\right)=b_{i}$, this implies that $\mathbf{w}_{0} \in \mathcal{X}$. Furthermore, we have the following conclusions.

Theorem 4.3 For any $\mathrm{x} \in \mathcal{X}, \mathrm{x} \wedge \mathrm{w}_{0} \in \mathcal{X}$.

Proof. Suppose that $\mathbf{x}=\left(x_{j}\right)_{j \in J}^{T} \in \mathcal{X}$. Since for all $i \in I, \bigvee_{j \in J}\left(a_{i j} \wedge x_{j} \wedge\left(x_{j}^{*} \wedge \vee_{i \in I} a_{i j}\right)\right)=$ $\bigvee_{j \in J}\left(a_{i j} \wedge x_{j}\right)=b_{i}$, it follows that $\mathbf{x} \wedge \mathbf{w}_{0} \in \mathcal{X}$.

Theorem 4.4 For any $\mathbf{x} \in \mathcal{X}^{0}, \mathbf{x} \leqslant \mathbf{y}^{*^{\prime}}$.

Proof. According to Theorem 4.3, for any $\mathbf{x} \in \mathcal{X}^{0}$, we have $\mathbf{x} \wedge \mathbf{w}_{0} \in \mathcal{X}$, then $\mathbf{x} \wedge \mathbf{w}_{0}=\mathbf{x} \wedge \mathbf{x}^{*} \wedge \mathbf{y}^{*^{\prime}}=$ $\mathbf{x} \wedge \mathbf{y}^{*^{\prime}} \in \mathcal{X}$. Since $\mathbf{x} \wedge \mathbf{y}^{*^{\prime}} \leqslant \mathbf{x}$ and $\mathbf{x} \in \mathcal{X}^{0}$, this implies that $\mathbf{x} \wedge \mathbf{y}^{*^{\prime}}=\mathbf{x}$. Consequently, $\mathbf{x} \leqslant \mathbf{y}^{*^{\prime}}$.

Theorem 4.5 If $\mathbf{x} \in \mathcal{X}, \mathrm{x} \wedge \mathbf{y}^{*}=\mathbf{0}$, then there are no elements $\mathbf{x}^{\prime} \in \mathcal{X}, \mathbf{x}^{\prime} \neq \mathcal{X}$, and $\mathbf{y} \in \mathcal{Y}$ such that $\mathbf{x}=\mathbf{x}^{\prime} \vee \mathbf{y}$.

Proof. If there exist elements $\mathbf{x}^{\prime} \in \mathcal{X}, \mathbf{x}^{\prime} \neq \mathbf{x}$, and $\mathbf{y} \in \mathcal{Y}$ such that $\mathbf{x}=\mathbf{x}^{\prime} \vee \mathbf{y}$, then $\mathbf{y}<\mathbf{x}$, and $\mathbf{y} \leqslant \mathbf{x} \wedge \mathbf{y}^{*}=\mathbf{0}$. Hence $\mathbf{y}=\mathbf{0}$. Therefore, $\mathbf{x}=\mathbf{x}^{\prime}$, a contradiction.

Theorem 4.6 If $\mathrm{x} \in \mathcal{X}$, then $\mathrm{x} \wedge \mathrm{y}^{*}=\mathbf{0}$ if and only if $\mathbf{x} \leqslant \mathbf{w}_{0}$.

Proof. If $\mathrm{x} \wedge \mathrm{y}^{*}=\mathbf{0}$, then $\mathrm{x}=\mathrm{x} \wedge \mathbf{1}=\mathrm{x} \wedge$ $\left(\mathbf{y}^{*} \vee \mathbf{y}^{*^{\prime}}\right)=\left(\mathbf{x} \wedge \mathbf{y}^{*}\right) \vee\left(\mathbf{x} \wedge \mathbf{y}^{*^{\prime}}\right)=\mathbf{0} \vee\left(\mathbf{x} \wedge \mathbf{y}^{*^{\prime}}\right)=$ $\mathbf{x} \wedge \mathbf{y}^{*^{\prime}}$. Thus $\mathbf{x} \leqslant \mathbf{y}^{*^{\prime}}$. Hence $\mathbf{x} \leqslant \mathbf{x}^{*} \wedge \mathbf{y}^{*^{\prime}}=\mathbf{w}_{0}$. Conversely, suppose that $\mathbf{x} \leqslant \mathbf{w}_{0}$, then $\mathbf{x} \wedge \mathbf{y}^{*} \leqslant$ $\mathbf{w}_{0} \wedge \mathbf{y}^{*}=\left(\mathbf{x}^{*} \wedge \mathbf{y}^{*^{\prime}}\right) \wedge \mathbf{y}^{*}=\mathbf{y}^{*^{\prime}} \wedge \mathbf{y}^{*}=\mathbf{0}$. Therefore, $\mathbf{x} \wedge \mathbf{y}^{*}=\mathbf{0}$.

Theorem 4.7 Let $\mathbf{x} \in \mathcal{X}$. If $\mathbf{x} \geqslant \mathbf{w}_{0}$, then there is an element $\mathbf{y} \in \mathcal{Y}$ such that $\mathbf{x}=\mathbf{w}_{0} \vee \mathbf{y}$.

Proof. Suppose that $\mathbf{x} \in \mathcal{X}$ and $\mathbf{x} \geqslant \mathbf{w}_{0}$. Let $\mathbf{y}=\mathbf{x} \wedge \mathbf{y}^{*}$. Then $\mathbf{y} \in \mathcal{Y}$, and $\mathbf{w}_{0} \vee \mathbf{y}=\mathbf{w}_{0} \vee(\mathbf{x} \wedge$ $\left.\mathbf{y}^{*}\right)=\left(\mathbf{w}_{0} \vee \mathbf{x}\right) \wedge\left(\mathbf{w}_{0} \vee \mathbf{y}^{*}\right)=\mathbf{x} \wedge\left(\left(\mathbf{x}^{*} \wedge \mathbf{y}^{*^{\prime}}\right) \vee \mathbf{y}^{*}\right)=$ $\mathbf{x} \wedge\left(\left(\mathbf{x}^{*} \vee \mathbf{y}^{*}\right) \wedge\left(\mathbf{y}^{*^{\prime}} \vee \mathbf{y}^{*}\right)\right)=\mathbf{x} \wedge\left(\mathbf{x}^{*} \wedge \mathbf{1}\right)=\mathbf{x}$

Remark 4.2 Let $\mathcal{X}_{0}=\left\{\mathbf{x} \in \mathcal{X}: \mathbf{x} \geqslant \mathbf{w}_{0}\right\} . B y$ Theorems 4.1 and 4.7, we have $\mathcal{X}_{0}=\left\{\mathbf{w}_{0}+y_{1} \mathbf{e}_{1}+\right.$ $\left.y_{2} \mathbf{e}_{2}+\cdots+y_{n} \mathbf{e}_{n}:\left(y_{1}, y_{2}, \cdots, y_{n}\right)^{T} \in \mathcal{Y}\right\}$. But according to Theorems 4.5 and 4.6, if $\mathrm{x} \in \mathcal{X}$, and $\mathbf{x}<\mathbf{w}_{0}$, then $\mathbf{x}$ can be represented only by the combination of itself and zero element. 
Example 4.1 Let $L$ be the lattice of power set of set $A=\{1,2,3,4\}$. Denote $1=A, 0=\emptyset$, $a_{1}=\{1,2,3\}, a_{2}=\{1,2,4\}, a_{3}=\{1,3,4\}, a_{4}=$ $\{2,3,4\}, b_{1}=\{1,2\}, b_{2}=\{1,3\}, b_{3}=\{1,4\}$, $b_{4}=\{2,3\}, b_{5}=\{2,4\}, b_{6}=\{3,4\}, c_{1}=\{1\}$, $c_{2}=\{2\}, c_{3}=\{3\}, c_{4}=\{4\}$. Consider equation $A \odot \mathbf{x}=\mathbf{b}$, where

$$
A=\left(\begin{array}{llll}
c_{1} & b_{3} & b_{4} & b_{1} \\
b_{2} & b_{1} & b_{5} & b_{6} \\
a_{1} & b_{5} & c_{4} & b_{3}
\end{array}\right)
$$

$\mathbf{b}=\left(c_{1}, b_{2}, a_{1}\right)^{T}$. Obviously, $\mathcal{X} \neq \emptyset$, and $\mathbf{x}^{*}=$ $\left(1, b_{2}, c_{1}, b_{2}\right)^{T}, \mathcal{X}^{0}=\left\{\mathbf{x}_{* 1}=\left(a_{1}, 0,0,0\right)^{T}, \mathbf{x}_{* 2}=\right.$ $\left.\left(b_{4}, c_{1}, 0, c_{1}\right)^{T}\right\}$. The associated equation is $A \odot \mathbf{x}=$ $\mathbf{0}$, and $\mathbf{y}^{*}=\left(c_{4}, c_{3}, c_{1}, 0\right)^{T}, \mathbf{e}_{1}=\left(c_{4}, 0,0,0\right)^{T}, \mathbf{e}_{2}=$ $\left(0, c_{3}, 0,0\right)^{T}, \mathbf{e}_{3}=\left(0,0, c_{1}, 0\right)^{T}, \mathbf{e}_{4}=(0,0,0,0)^{T}$. Consider a solution $\mathbf{x}_{1}=\left(a_{1}, c_{1}, c_{1}, 0\right)^{T} \in \mathcal{X}, s$ ince $\mathbf{y}^{*} \wedge \mathbf{x}=\left(0,0, c_{1}, 0\right)^{T}$, we have $\mathbf{x}_{1}=\mathbf{w}+$ $0 \mathbf{e}_{1}+0 \mathbf{e}_{2}+c_{1} \mathbf{e}_{3}$, where $\mathbf{w}=\left(a_{1}, c_{1}, 0,0\right)^{T} \in \mathcal{X}$. For another solution $\mathbf{x}_{2}=\left(a_{1}, c_{1}, c_{1}, b_{2}\right)^{T} \in \mathcal{X}, s$ ince $\mathbf{w}_{0}=\left(a_{1}, c_{1}, 0, b_{2}\right)^{T}$, and $\mathbf{x}_{2} \geqslant \mathbf{w}_{0}$, we have $\mathbf{x}_{2}=\mathbf{w}_{0} \vee \mathbf{y}$, where $\mathbf{y}=\left(0,0, c_{1}, 0\right)^{T} \in \mathcal{Y}$, that is, $\mathbf{x}_{2}=\mathbf{w}_{0}+0 \mathbf{e}_{1}+0 \mathbf{e}_{2}+c_{1} \mathbf{e}_{3}$. And these also demonstrate the description in Remark 4.1.

Now we consider a special case of Eq.(1), that is, $|I|=1$, and assume that the equation is

$$
\bigvee_{j \in J}\left(a_{j} \wedge x_{j}\right)=b
$$

and its associated equation is

$$
\bigvee_{j \in J}\left(a_{j} \wedge x_{j}\right)=0 .
$$

Obviously, the greatest solution of Eq.(4) is $\mathbf{y}^{*}=$ $\left(a_{j}^{\prime}\right)_{j \in J}^{T}$, and $\mathbf{e}_{k}=\left(y_{j}\right)_{j \in J}^{T}$, where $y_{k}=a_{k}^{\prime}$ and $y_{j}=$ 0 if $j \neq k$.

There are some relationships between the minimal solution of Eq.(3) and the minimal decomposition of $b$.

Theorem 4.8 Let $J^{\prime} \subseteq J$. If $M=\left\{s_{j} \in L: s_{j} \leqslant\right.$ $a_{j}$ for each $\left.j \in J^{\prime}\right\} \in \mathcal{M}_{b}$, then $\mathbf{x}_{*}=\left(x_{j *}\right)_{j \in J}^{T} \in$ $\mathcal{X}^{0}$, where $x_{j *}=s_{j}$ if $j \in J^{\prime}$ and $x_{j *}=0$ if $j \in$ $J-J^{\prime}$.

Proof. It is clear that $\mathbf{x}_{*}=\left(x_{j *}\right)_{j \in J}^{T} \in \mathcal{X}$. If $\mathbf{x}=\left(x_{j}\right)_{j \in J}^{T} \in \mathcal{X}$ and $\mathbf{x} \leqslant \mathbf{x}_{*}$, then $x_{j} \leqslant x_{j *}$ for all $j \in J$. This implies that $x_{j} \leqslant x_{j *}$ if $j \in J^{\prime}$ and $x_{j}=x_{j *}=0$ if $j \in J-J^{\prime}$. Thus $b=\bigvee_{j \in J^{\prime}} x_{j}$. This last formula combined with the fact $M=\left\{s_{j} \in L\right.$ : $\left.j \in J^{\prime}\right\} \in \mathcal{M}_{b}$ yields that $x_{j}=s_{j}=x_{j *}$ for all $j \in J$. Consequently, $\mathbf{x}_{*}=\left(x_{j *}\right)_{j \in J}^{T} \in \mathcal{X}^{0}$.

Theorem 4.9 If $\mathcal{X} \neq \emptyset$, then for any $\mathbf{x} \in \mathcal{X}$, there is some $\mathbf{x}_{*} \in \mathcal{X}^{0}$ such that $\mathbf{x}_{*} \leqslant \mathbf{x}$.

Proof. Suppose that $\mathbf{x}=\left(x_{j}\right)_{j \in J}^{T} \in \mathcal{X}$. Then $\bigvee_{j \in J}\left(a_{j} \wedge x_{j}\right)=b$. In this representation, Removing the redundant elements, we can get a irredundant decomposition $b=\bigvee_{j \in J^{\prime}}\left(a_{j} \wedge x_{j}\right)$, where $J^{\prime} \subseteq J$ and $b \neq \bigvee_{j \in J^{\prime}, j \neq k}\left(a_{j} \wedge x_{j}\right)$ for each $k \in J^{\prime}$. By Theorem 3.3, there exists $M=\left\{s_{j} \in L: j \in J^{\prime}\right\} \in$ $\mathcal{M}_{b}$ such that $s_{j} \leqslant a_{j} \wedge x_{j}$ for all $j \in J^{\prime}$. Define $\mathbf{x}_{*}=\left(x_{j *}\right)_{j \in J}^{T}$ with $x_{j *}=a_{j} \wedge x_{j}$ if $j \in J^{\prime}$ and $x_{j *}=0$ if $j \in J-J^{\prime}$. It is clear that $\mathbf{x}_{*} \leqslant \mathbf{x}$ and $\mathbf{x}_{*} \in \mathcal{X}^{0}$.

According to Theorem 4.9, if $\mathcal{X} \neq \emptyset$, then $\mathcal{X}^{0} \neq \emptyset$. Now let $\mathbf{x}_{0}=\bigvee_{\mathbf{x} \in \mathcal{X}^{0}} \mathbf{x}$. Then we have the following statement:

Theorem 4.10 $\mathbf{x}_{0}=\left(a_{j} \wedge b\right)_{j \in J}$ and $\mathbf{y}^{*} \vee \mathbf{x}_{0}=\mathbf{x}^{*}$.

Proof. Let $\mathbf{x}_{0}=\left(x_{j 0}\right)_{j \in J}^{T}$. By Lemma 2.6, for any $\mathbf{x}_{*}=\left(x_{j *}\right)_{j \in J}^{T} \in \mathcal{X}^{0}$, we have $x_{j *} \leqslant a_{j} \wedge b$ for all $j \in J$. Then $x_{j 0} \leqslant a_{j} \wedge b$ for all $j \in J$. In the following, we show that for any $k \in J$, there is some $\mathbf{x}_{*}=\left(x_{j *}\right)_{j \in J}^{T} \in \mathcal{X}^{0}$ such that $x_{k *}=a_{k} \wedge b$. Obviously, if $a_{k} \wedge b=0$, then $x_{k *}=0$ for any $\mathbf{x}_{*}=$ $\left(x_{j *}\right)_{j \in J}^{T} \in \mathcal{X}^{0}$. If $a_{k} \wedge b=b$, that is, $a_{k} \geqslant b$, then $\mathbf{x}_{*}=\left(x_{j *}\right)_{j \in J}^{T} \in \mathcal{X}^{0}$, where $x_{k *}=b$ and $x_{j *}=0$ with $j \neq k$. If $0<a_{k} \wedge b<b$, then $a_{k} \wedge b \in D(b)$. Since $b=\bigvee_{j \in J}\left(a_{j} \wedge b\right)=\left(a_{k} \wedge b\right) \vee \bigvee_{j \in J, j \neq k}\left(a_{j} \wedge b\right)$, by Theorem 3.4 it follows that there is a minimal decomposition $b=\left(a_{k} \wedge b\right) \vee \bigvee_{j \in J_{k}} u_{j}$, where $J_{k} \subseteq$ $J-\{k\}$ and $u_{j} \leqslant a_{j} \wedge b$ for all $j \in J_{k}$. Define $\mathbf{x}_{*}=\left(x_{j *}\right)_{j \in J}^{T}$ with $x_{k *}=a_{k} \wedge b, x_{j *}=u_{j}$ if $j \in J_{k}$ and $x_{j *}=0$ if $j \in J-\left(J_{k} \cup\{k\}\right)$. Then $\mathbf{x}_{*} \in \mathcal{X}^{0}$ by Theorem 4.8. With the above discussions, we have $\mathbf{x}_{0}=\left(a_{j} \wedge b\right)_{j \in J}^{T}$. Now for any $j \in J, x_{j}^{*}=a_{j}^{\prime} \vee b$ and $y_{j}^{*}=a_{j}^{\prime}$, it follows that for all $j \in J, a_{j}^{\prime} \vee\left(a_{j} \wedge b\right)=$ $\left(a_{j}^{\prime} \vee a_{j}\right) \wedge\left(a_{j}^{\prime} \vee b\right)=1 \wedge\left(a_{j}^{\prime} \vee b\right)=a_{j}^{\prime} \vee b=x_{j}^{*}$. Consequently, $\mathbf{y}^{*} \vee \mathbf{x}_{0}=\mathbf{x}^{*}$.

Theorem 4.11 Let $\mathrm{x} \in \mathcal{X}$, and let $\mathrm{x}_{1}=$ $\bigvee_{\mathbf{x}_{*} \in \mathcal{X}^{0}, \mathbf{x}_{*} \leqslant \mathbf{x}} \mathbf{x}_{*}$. Then there is some element $\mathbf{y} \in \mathcal{Y}$ such that $\mathbf{x}=\mathbf{x}_{1} \vee \mathbf{y}$.

Proof. Let $\mathbf{x}=\left(x_{j}\right)_{j \in J}^{T} \in \mathcal{X}$ and $\mathbf{x}_{1}=\left(x_{j 1}\right)_{j \in J}^{T}$. Then $x_{j 1} \leqslant a_{j}$ and $x_{j 1} \leqslant x_{j}$, that is, $x_{j 1} \leqslant a_{j} \wedge x_{j}$. In the following, we show that $x_{j 1}=a_{j} \wedge x_{j}$ for all $j \in J$. It is clear that we only show that for any $k \in J$, there is some $\mathbf{x}_{*}=\left(x_{j *}\right)_{j \in J}^{T} \in \mathcal{X}^{0}$ such that $\mathbf{x}_{*} \leqslant \mathbf{x}$ and $x_{k *}=a_{k} \wedge x_{k}$. Obviously, if $a_{k} \wedge x_{k}=0$, then $x_{k *}=0$ for any $\mathbf{x}_{*}=\left(x_{j *}\right)_{j \in J}^{T} \in \mathcal{X}^{0}$ with $\mathbf{x}_{*} \leqslant \mathbf{x}$. If $a_{k} \wedge x_{k}=b$, that is, $a_{k} \geqslant b$, then $\mathbf{x}_{*}=$ $\left(x_{j *}\right)_{j \in J}^{T} \in \mathcal{X}^{0}$, where $x_{k *}=b$ and $x_{j *}=0$ with $j \neq$ $k$. If $0<a_{k} \wedge x_{k}<b$, then $a_{k} \wedge x_{k} \in D(b)$. Similar to the proof of Theorem 4.10, there is a minimal solution $\mathbf{x}_{*}=\left(x_{j *}\right)_{j \in J} \in \mathcal{X}^{0}$ such that $\mathbf{x}_{*} \leqslant \mathbf{x}$ and $x_{k *}=a_{k} \wedge x_{k}$. With the above discussions, we have $\mathbf{x}_{1}=\left(a_{j} \wedge x_{j}\right)_{j \in J}^{T}$. Now let $\mathbf{y}=\mathbf{y}^{*} \wedge \mathbf{x}$, then $\mathbf{y} \in \mathcal{Y}$. Furthermore, for every $j \in J,\left(a_{j} \wedge x_{j}\right) \vee\left(a_{j}^{\prime} \wedge x_{j}\right)=$ $\left(a_{j} \vee a_{j}^{\prime}\right) \wedge x_{j}=1 \wedge x_{j}=x_{j}$, that is, $\mathbf{x}=\mathbf{x}_{1} \vee \mathbf{y}$.

In particular, we have the following statements.

Corollary 4.1 For any $\mathrm{x} \in \mathcal{X}$, if $\mathrm{x}_{0} \leqslant \mathrm{x} \leqslant \mathrm{x}^{*}$, then there is a solution $\mathbf{y} \in \mathcal{Y}$ such that $\mathbf{x}=\mathbf{x}_{0} \vee \mathbf{y}$. 
Corollary 4.2 If $\mathcal{X}$ has the least solution $\mathbf{x}_{*}$, then for any $\mathbf{x} \in \mathcal{X}$, there is a solution $\mathbf{y} \in \mathcal{Y}$ such that $\mathbf{x}=\mathbf{x}_{*} \vee \mathbf{y}$.

Remark 4.3 According to Theorem 4.11, we have that for any $\mathbf{x}=\left(x_{j}\right)_{j \in J}^{T} \in \mathcal{X}$ and $\mathbf{x}_{1} \leqslant \mathbf{x} \leqslant \mathbf{x}^{*}$, $\mathbf{x}$ can be represented by a linear combination of a special solution $\mathbf{x}_{1}$ of $\mathcal{X}$ and some certain solutions of $\mathcal{Y}$, that is, $\mathbf{x}=\mathbf{x}_{1}+y_{1} \mathbf{e}_{1}+y_{2} \mathbf{e}_{2}+\cdots+y_{n} \mathbf{e}_{n}$, where $\mathbf{y}=\mathbf{y}^{*} \wedge \mathbf{x}=\left(y_{j}\right)_{j \in J}^{T} \in \mathcal{Y}$.

Example 4.2 Let $L$ be the lattice from Example 4.1. Consider equation $\left(c_{1} \wedge x_{1}\right) \vee\left(c_{2} \wedge x_{2}\right) \vee$ $\left(b_{4} \wedge x_{3}\right) \vee\left(a_{1} \wedge x_{4}\right) \vee\left(a_{2} \wedge x_{5}\right)=b_{5}$. Obviously, $\mathcal{X} \neq \emptyset$, and $\mathbf{x}^{*}=\left(a_{4}, 1, a_{2}, b_{5}, a_{4}\right)^{T}, \mathcal{X}^{0}=$ $\left\{\mathbf{x}_{* 1}=\left(0,0,0,0, b_{5}\right)^{T}, \mathbf{x}_{* 2}=\left(0, c_{2}, 0,0, c_{4}\right)^{T}, \mathbf{x}_{* 3}=\right.$ $\left.\left(0,0, c_{2}, 0, c_{4}\right)^{T}, \mathbf{x}_{* 4}=\left(0,0,0, c_{2}, c_{4}\right)^{T}\right\}$. The associated equation is $\left(c_{1} \wedge x_{1}\right) \vee\left(c_{2} \wedge x_{2}\right) \vee$ $\left(b_{4} \wedge x_{3}\right) \vee\left(a_{1} \wedge x_{4}\right) \vee\left(a_{2} \wedge x_{5}\right)=0$, and $\mathbf{y}^{*}=\left(a_{4}, a_{3}, b_{3}, c_{4}, c_{3}\right)^{T}, \quad \mathbf{e}_{1}=\left(a_{4}, 0,0,0,0\right)^{T}$, $\mathbf{e}_{2}=\left(0, a_{3}, 0,0,0\right)^{T}, \mathbf{e}_{3}=\left(0,0, b_{3}, 0,0\right)^{T}, \mathbf{e}_{4}=$ $\left(0,0,0, c_{4}, 0\right)^{T}, \mathbf{e}_{5}=\left(0,0,0,0, c_{3}\right)^{T}$. Consider $a$ solution $\mathbf{x}=\left(b_{4}, a_{1}, b_{1}, 0, b_{6}\right)^{T} \in \mathcal{X}$, since $\mathbf{x} \geqslant$ $\mathbf{x}_{* 2} \vee \mathbf{x}_{* 3}=\left(0, c_{2}, c_{2}, 0, c_{4}\right)=\mathbf{x}_{1}, \mathbf{y}^{*} \wedge \mathbf{x}=$ $\left(b_{4}, b_{2}, c_{1}, 0, c_{3}\right)^{T}$, it follows that $\mathbf{x}=\mathbf{x}_{1}+b_{4} \mathbf{e}_{1}+$ $b_{2} \mathbf{e}_{2}+c_{1} \mathbf{e}_{3}+0 \mathbf{e}_{4}+c_{3} \mathbf{e}_{5}$.

\section{Conclusions}

In this contribution, we investigated the structure of the solution set of Eq.(1) over complete Boolean algebras similar to linear algebraic systems, and showed that each solution can be represented by a linear combination of a special solution of Eq.(1) and some certain solutions of the homogeneous equations associated with Eq. (1). In Eq.(3), the special solution can be determined by the minimal solutions. Unfortunately, without additional conditions, for Eq.(1), the special solution is not unique.

\section{Acknowledgments}

This work is supported by National Natural Science Foundation of China (Nos.11171242 and 11201325), the Basic Research Programs of Sichuan Province of China (No.2011JY0133) and the Scientific Research Fund of Sichuan Provincial Education Department in China(No.13ZB0110).

\section{References}

[1] E. Bartl, R. Belohlavek, V. Vychodil, Bivalent and other solutions of fuzzy relational equations via linguistic hedges, Fuzzy Sets and Systems 187 (2012) 103-112.

[2] G. Birkhoff, Lattice Theory, Vol.XXV, 3rd ed., American Mathematical Society Colloquium Publications, Providence, RI, 1979.

[3] P. Crawley, R. P. Dilworth, Algebraic Theory of Lattices, Prentice-Hall, Englewood Cliffs, NJ, 1973.
[4] B. De Baets, An order-theoretic approach to solving sup-T equations, in: D. Ruan (Ed.), Fuzzy Set Theory and Advanced Mathematical Applications, Kluwer Academic Publishers, Dordrecht, 1995, pp. 67-87.

[5] J. C. Diaz-Moreno, J. Medina, Using concep$\mathrm{t}$ lattice theory to obtain the set of solutions of multi-adjoint relation equations, Information Sciences 266( 2014) 218-225.

[6] A. Di Nola, S. Sessa, W. Pedrycz and E. Sanchez, Fuzzy Relation Equations and Their Applications to Knowledge Engineering, Kluwer Academic Publishers, Dordrecht, Boston/London, 1989.

[7] A. Di Nola, A.Lettieri, I.Perfilieva, V.Novák, Algebraic analysis of fuzzy systems, Fuzzy Sets and Systems 158 (2007) 1-22.

[8] J. Drewniak, Z. Matusiewicz, Properties of max* fuzzy relation equations, Soft Computing 14 (2010) 1037-1041.

[9] R.L. Goodstein, Boolean Algebra, Pergamon Press, Oxford; MacMillan Co., New York, 1963.

[10] Song-Chol Han, Hong-Xing Li, Jia-Yin Wang, Resolution of matrix equations over arbitrary Brouwerian lattices, Fuzzy Sets and Systems 159 (2008) 40-46.

[11] M. Higashi, G. J. Klir, Resolution of finite fuzzy relation equations, Fuzzy Sets and Systems 13(1984) 65-82.

[12] K. Hirota, W. Pedrycz, Data compression with fuzzy relational equations, Fuzzy Sets and Systems 126 (2002) 325-335.

[13] V.Loia, S.Sessa, Fuzzy relation equations for coding/decoding processes of images and videos, Information Sciences 171 (2005) 145-172.

[14] J. Medina, E. Turunen, E. Bartl, J. C. D“a azMoreno, Minimal Solutions of Fuzzy Relation Equations with General Operators on the Unit Interval,Information Processing and Managemen$\mathrm{t}$ of Uncertainty in Knowledge-Based Systems Communications in Computer and Information Science, Montpellier, France, 2014, pp. 15-19.

[15] W. Pedrycz, A. V. Vasilakos, Modularization of fuzzy relational equations, Soft Computing 6 (2002) 33-37.

[16] K. Peeva, Y. Kyosev, Algorithm for Solving Max-product Fuzzy Relational Equations, Soft Computing 11 (2007) 593-605.

[17] K. Peeva, Resolution of fuzzy relational equations-methods,algorithm and software with applications, Information Sciences 234 (2013) 44-63.

[18] I.Perfilieva, Semi-linear spaces, in: H.Noguchi, H.Ishii, et al.(Eds.), Proceeding of Seventh Czech-Japanese Seminar on Data Analysis and Decision Making under Uncertainty, Hyogo, Japan, 2004, pp.127-130.

[19] I. Perfilieva, Finitary solvability conditions for systems of fuzzy relation equations, Information Sciences 234 (2013) 29-43. 
[20] Xiao-bing Qu, Xue-ping Wang, Some properties of infinite fuzzy relational equations on complete Brouwerian lattices, Fuzzy Sets and Systems 158 (2007) $1327-1339$.

[21] E. Sanchez, Resolution of composite fuzzy relation equations, Information and Control 30 (1976) 38-48.

[22] B.S.Shieh, Deriving minimal solutions for fuzzy relation equations with max-product composition, Information Sciences 178(2008) 3766-3774.

[23] Feng Sun, Xue-ping Wang, Xiao-bing Qu, Minimal join decompositions and their application$\mathrm{s}$ to fuzzy relation equations over complete Brouwerian lattices, Information Sciences 224 (2013) 143-151.

[24] Xue-Ping Wang, Method of solution to fuzzy relation equations in a complete Brouwerian lattice, Fuzzy sets and systems 120 (2001) 409-414.

[25] Xue-Ping Wang, Infinite fuzzy relational equations on a complete Brouwerian lattice, Fuzzy Sets and Systems 138 (2003) 657-666.

[26] Xue-ping Wang, Shan Zhao, Solution sets of finite fuzzy relation equations with sup-inf composition over bounded Brouwerian lattices, Information Sciences 234 (2013) 80-85.

[27] Cui-kui Zhao, On matrix equations in a class of complete and completely distributive lattice, Fuzzy Sets and Systems 22 (1987) 303-320. 